\title{
Phenotypes of a family with XLH with a novel PHEX mutation
}

\author{
Akiko Yamamoto $\mathbb{D}^{1}$, Toshiro Nakamura', Yasuhisa Ohata $\mathbb{D}^{2}$, Takuo Kubota ${ }^{2}$ and Keiichi Ozono²
}

\begin{abstract}
$X$-linked hypophosphatemia $(\mathrm{XLH})$ is the most common form of heritable hypophosphatemic rickets. We encountered a 4-year-old boy with a novel variant in the phosphate-regulating neutral endopeptidase homolog X-linked (PHEX) gene who presented with a short stature, genu valgum, and scaphocephaly. The same mutation was identified in his mother and sister; however, the patient presented with a more severe case.
\end{abstract}

$\mathrm{X}$-linked hypophosphatemia (XLH) is an X-linked dominant disorder and the most common form of heritable rickets, with a case rate estimate of $\sim 1$ case per 20,000 live births ${ }^{1}$. Inactive mutations in PHEX, which is located in $\mathrm{Xp} 22.1-22.2$, have been implicated in the pathogenesis of XLH. More than 200 different mutations in PHEX have been identified to date ${ }^{2}$. XLH is characterized by rickets accompanied by bone deformities, a short stature, dental anomalies, bone pain, hearing difficulties, enthesopathy, and muscular dysfunction ${ }^{3}$. Patients with XLH have hypophosphatemia with low levels of renal phosphate reabsorption, normal serum calcium levels, elevated serum alkaline phosphatase activities, normal or increased parathyroid hormone levels, normal or increased 1,25-dihydroxyvitamin D3 levels, and elevated FGF23 levels ${ }^{4}$. Marked variations in XLH phenotypes have been reported. We herein describe a boy and his family who had XLH and a novel splice-site mutation in PHEX.

A boy aged 4 years and 11 months presented with a short stature to the endocrinology unit of our hospital. He was born after a normal pregnancy, with a birth weight of $3130 \mathrm{~g}$ and body length of $48 \mathrm{~cm}$. He had an elder brother as well as a younger brother and sister. His mother and grandmother both had a short stature and genu valgum,

Correspondence: Akiko Yamamoto (a-yamamoto@kumachu.gr.jp)

${ }^{1}$ Department of Pediatrics, Kumamoto Chuo Hospital, Kumamoto, Japan

${ }^{2}$ Department of Pediatrics, Osaka University Graduate School of Medicine,

Osaka, Japan whereas his elder and younger brothers did not have a short stature (Fig. 1(a)).

At the time of his visit, his height was $95.4 \mathrm{~cm}$ $(-2.51$ standard deviation score (SDS)), weight was $14.8 \mathrm{~kg}(-1.13 \mathrm{SDS})$, and growth rate was $5.2 \mathrm{~cm}$ per year $(-1.40 \mathrm{SDS})$, and genu valgum and scaphocephaly were both evident. He did not have any dental issues or other symptoms.

His serum calcium level was $9.71 \mathrm{mg} / \mathrm{dl}$ (normal range: $8.8-10.3 \mathrm{mg} / \mathrm{dl}$ ), phosphate level was $2.4 \mathrm{mg} / \mathrm{dl}$ $(3.8-5.9 \mathrm{mg} / \mathrm{dl})$, alkaline phosphatase (ALP) level was $1175 \mathrm{IU} / \mathrm{L}(420-1200 \mathrm{mg} / \mathrm{dl})$, intact parathyroid hormone (PTH) (ECLIA) level was $73 \mathrm{pg} / \mathrm{ml}(10-65 \mathrm{pg} / \mathrm{ml})$, and $25(\mathrm{OH})$ vitamin D (RIA) level was $17 \mathrm{ng} / \mathrm{ml}$ $(>30 \mathrm{ng} / \mathrm{ml})$. He had a low \% tubular reabsorption of phosphorus (TRP) value (89.3\%) and a high intact FGF23 (ELISA) level $(73 \mathrm{pg} / \mathrm{ml})(16-69 \mathrm{pg} / \mathrm{ml})$. Bilateral femoral and tibial bowing with fraying and cupping of the metaphysis were noted on the X-ray images (Fig. 1b). Brain CT scans showed craniosynostosis (Fig. 1c) and a mild Chiari malformation.

Since XLH was suspected, a genetic analysis was performed, which was approved by the Institutional Review Boards of the participating institutions. Written informed consent was obtained from the study participants, including their consent to participate and permission to publish the findings obtained. Genomic DNA was extracted from whole blood samples using a magLEAD Consumable Kit (Precision System Science Co., Ltd., Chiba, Japan). The amplicons generated by PCR with the 


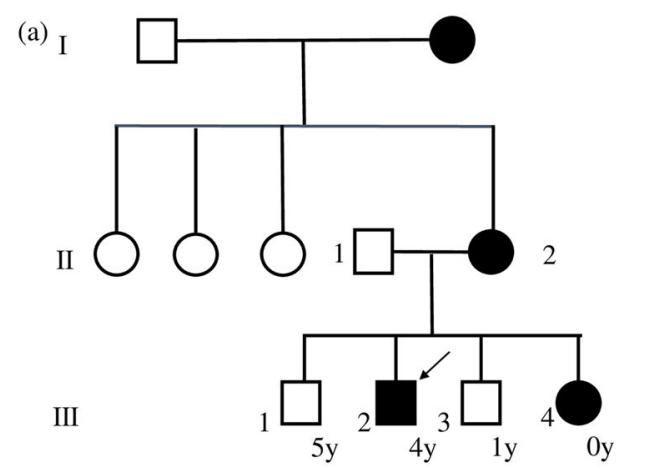

(b)

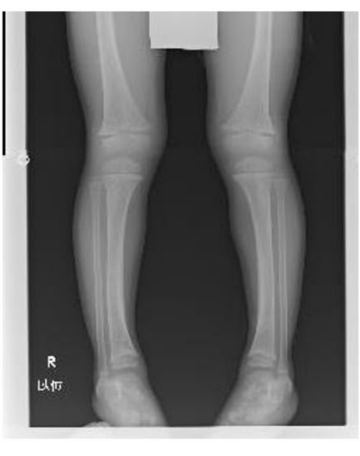

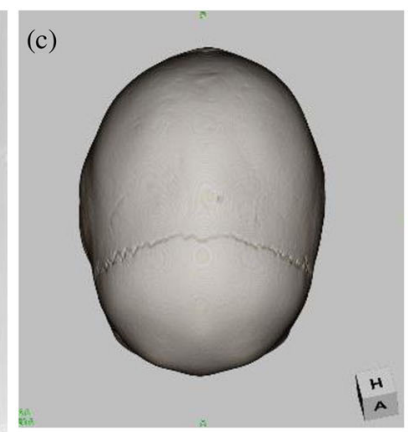

(d)
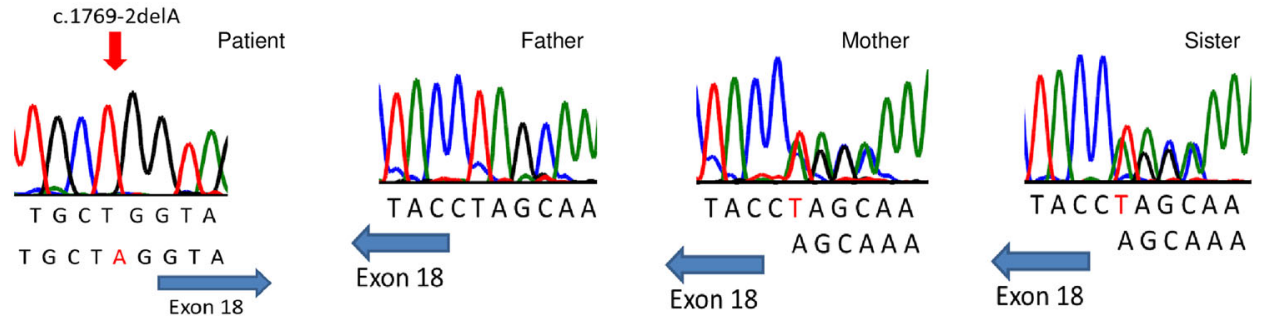

Fig. 1 Clinical characteristics and genetic findings in the patient. a A family tree: the solid square indicates the index case. Solid circles indicate family members who developed rickets. Open symbols indicate healthy members. b A radiological examination showing fraying and cupping of the metaphysis and bilateral leg deformation. c Three-dimensional CT showing skull bone window reconstruction. The complete closure of sagittal sutures. d Gene analysis. A novel splice acceptor site mutation, c.1769-2delA, was identified in PHEX. The same heterozygous mutation was detected in the mother and sister of the patient. No mutations were found in the father.

designed primers were Sanger sequenced using a 3730 DNA analyzer (Applied Biosystems, Foster City, CA, USA), which revealed a novel PHEX mutation in intron 17, NM_000444.6: c.1769-2delA. We analyzed this novel mutation by Human Splicing Finder 3.1 (http://www.umd. be/HSF3/index.html), and the results obtained suggested that this mutation led to a broken WT acceptor site and affected splicing. The mutation detected in this patient was not found in the Human Gene Mutation Database (http://www.hgmd.cf.ac.uk/ac/index.php) or Genome Aggregation Database (http://gnomad.broadinstitute.org).

The diagnosis of XLH was confirmed by a genetic analysis, and therapy comprising phosphate $55 \mathrm{mg} / \mathrm{kg} /$ day and alfacalcidol $35 \mathrm{ng} / \mathrm{kg} /$ day was initiated. DNA samples obtained from the patient's younger sister, mother, and father were also subjected to a genetic analysis. No abnormalities were detected in PHEX in the father, whereas the mother and sister had the same mutation. His mother and sister both had hypophosphatemia, elevated ALP enzyme activities, and high intact FGF23 levels (Table 1, Fig. 1d). His mother also had a short stature $(148 \mathrm{~cm})$ and genu valgum, whereas his sister only presented with mild genu valgum (her height was $-1.78 \mathrm{SD}$ ). Although we were unable to genetically analyze the DNA from his grandmother, she also had a short stature, genu valgum, and bone pain.

We herein described a novel variant mutation in the splice site of intron 17 in PHEX of a boy and his family with XLH. XLH is a dominant disorder and the most common form of heritable rickets, and inactive mutations in PHEX have been implicated in the pathogenesis of $\mathrm{XLH}$. In accordance with the present results, splice-site mutations have been shown to be responsible for $17 \%$ of all reported PHEX mutations ${ }^{5}$. Other mutations have also been reported in the splice acceptor site of intron $17^{2,6,7}$. Hue Yue et al. described a case of a 16-year-old boy with a PHEX gene mutation in the splice acceptor site of intron 17 (c. $1768+2 \mathrm{~T}>\mathrm{G}$ ). This was a sporadic case that was characterized by leg bowing, hip pain, and growth retardation. In contrast, the present case had a familial mutation in PHEX along with a short stature, genu valgum, and scaphocephaly. Scaphocephaly is a form of craniosynostosis, a cranial malformation caused by premature fusing of the sagittal suture, and may develop in infants as young as 1 year of age $\mathrm{e}^{3,8}$. Craniosynostosis occurs in $\sim 60 \%$ of children with $\mathrm{XLH}^{9}$. Between $25 \%$ and $50 \%$ of children with XLH also have a Chiari type 1 malformation, which causes the cerebellar tonsils to herniate through the foramen magnum ${ }^{9,10}$. The sister, mother, and grandmother of the patient in the present report did not have scaphocephaly. In a large case series, gender differences in the disease severity of XLH were not observed $^{7,11}$; however, the severity and clinical manifestations of XLH patients varied markedly within the same family ${ }^{12}$. Among the family members tested, only the boy presented with sagittal synostosis and a mild Chiari malformation. Congenital forms of craniosynostosis 


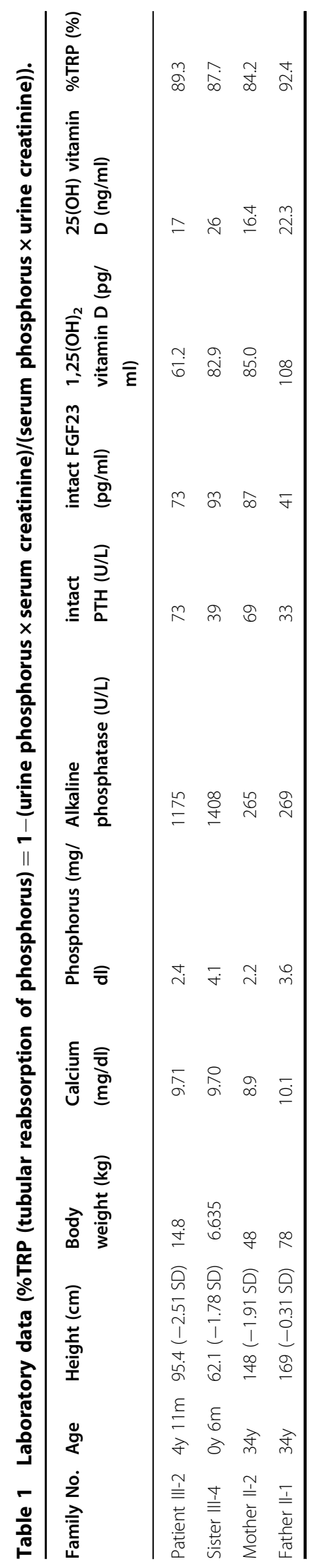

mostly develop in infancy, whereas those caused by metabolic disorders are more common in infants aged $\sim 2$ years ${ }^{10}$. Therefore, the younger sister was followed up for symptoms of craniosynostosis.

In summary, we herein described the novel splicing of and a familial mutation in PHEX. An early diagnosis facilitates the rapid initiation of proper treatment. Since we detected the same mutation in the younger sister of the patient in our report, she underwent regular followups. We will also carefully monitor symptoms and disease progression in this family in the future.

\section{HGV database}

The relevant data from this Data Report are hosted at the Human Genome Variation Database at https://doi.org/10.6084/m9.figshare.hgv.2823.

\section{Acknowledgements}

We thank the family for allowing us to report this case.

\section{Conflict of interest}

The authors declare that they have no conflict of interest.

\section{Publisher's note}

Springer Nature remains neutral with regard to jurisdictional claims in published maps and institutional affiliations.

Received: 20 January 2020 Revised: 2 March 2020 Accepted: 3 March 2020. Published online: 31 March 2020

\section{References}

1. Sako, S. et al. A novel PHEX mutation associated with vitamin D-resistant rickets. Hum. Genome Var 6, 9 (2016).

2. Ruppe, M. D. et al. Mutational analysis of PHEX, FGF23 and DMP1 in a cohort of patients with hypophosphatemic rickets. Clin. Endocrinol 74, 312-318 (2011).

3. Beck-Nielsen, S. S. et al. FGF23 and its role in X-linked hypophosphatemiarelated morbidity. Orphanet J. Rare Dis. 14, 58 (2019).

4. Cheon, C. K. et al. A novel de novo mutation within PHEX gene in a young girl with hypophosphatemic rickets and review of literature. Ann. Pediatr. Endocrinol. Metab. 19, 36 (2014).

5. BinEssa, H. A. et al. Functional analysis of 22 splice-site mutations in the PHEX, the causative gene in $\mathrm{X}$-linked dominant hypophosphatemic rickets. Bone 125, 186-193 (2019).

6. Yue, $\mathrm{H}$. et al. Identification of two novel mutations in the PHEX gene in Chinese patients with hypophosphatemic rickets/osteomalacia. PLOS ONE 9, e97830 (2014).

7. Zhang, $C$. et al. Clinical and genetic analysis in a large Chinese cohort of patients with X-linked hypophosphatemia. Bone 121, 212-220 (2019).

8. Haffner, D. et al. Clinical practice recommendations for the diagnosis and management of X-linked hypophosphataemia. Nat. Rev. Nephrol 15, 435-455 (2019).

9. Rothenbuhler, A. et al. High incidence of cranial synostosis and Chiari I malformation in children with X-linked hypophosphatemic rickets (XLHR). J. Bone Min. Res. 34, 490-496 (2019).

10. Vega, R. A. et al. Hypophosphatemic rickets and craniosynostosis: a multicenter case series. J. Neurosurg. Pediatr 17, 1-7 (2016).

11. Whyte, M. P., Schranck, F. W. \& Armamento-Villareal, R. X-linked hypophosphatemia: a search for gender, race, anticipation, or parent of origin effects on disease expression in children. J. Clin. Endocrinol. Metab. 81, 4075-4080 (1996).

12. Stickler, G. B., Beabout, J. W. \& Riggs, B. L. Vitamin D-resistant rickets: clinical experience with 41 typical familial hypophosphatemic patients and 2 atypical nonfamilial cases. Mayo Clin. Proc. 45, 197-218 (1970). 